\title{
Network Intrusion Prediction Model based on RBF Features Classification
}

\author{
Wang Xing-zhu ${ }^{1}$ \\ 1. Furong College Hunan University of Arts and Science Hunan Changde, \\ 415000, China \\ Wangxzhu@sina.com
}

\begin{abstract}
According to the relationship between feature subset and parameters of RBF neural network, in order to improve the intrusion detection accuracy, it proposed an improved particle swarm optimization neural network of network intrusion detection model. Network feature subset and parameters of RBF neural network were regarded as a particle, through collaboration and information exchange between particles to find the optimal feature subset and parameters of RBF neural network, so as to establish the optimal network intrusion detection model, and using KDD Cup 99 data sets to carry out simulation experiment. The simulation results showed that, IPSO-RBF neural network reduced the feature dimensions, and the better parameters of RBF neural network was obtained then, which is a kind of network intrusion detection model with high detection accuracy and high speed.
\end{abstract}

Keywords: Network intrusion; Particle swarm algorithm; Neural network; Feature selection

\section{Introduction}

As the network user is increasing, network attack is becoming more and more frequent, it is very difficult for the passive, traditional firewall technology to meet the requirement of network security, while network intrusion detection system (IDS) is a kind of initiative precautionary technology, has become an important topic in the field of network security [1].

Network intrusion detection is the classification problem in pattern recognition, it mainly includes modules such as the feature selection and classifier selection and optimization, etc., the network data is very complex with characteristics of high dimension, and feature set contains some redundant features and useless features, these will increase the training time model and the computational complexity, and has adverse effects on the result of the intrusion detection [2].Therefore, before the network intrusion detection modeling, it is often conduct feature selection algorithm to select feature subsets for detection results, and reduce feature dimensions, the current methods based on such as sequence search algorithms, principal component analysis, the genetic algorithm, particle swarm optimization algorithm and other feature selection methods [3-5].In addition to feature subset, network intrusion detection results are also closely related to classifier and parameters, the current network intrusion detection model mainly include nonlinear classification algorithms as Bayesian network, neural network and support vector machine (SVM) [7-9]. Due to network intrusion detection is a system large sample classification problem, for such large sample classification problem, the training speed of support vector machine (SVM) is slow, which is not good for real-time and linear of network intrusion detection. Neural networks do not require a priori knowledge, but can make nonlinear, infinite approximation to system, especially the RBF neural network has some 
advantages such as simple structure, fast learning, has been widely used in network intrusion detection [10]. In practical applications, the RBF neural network is closely related to the classification performance and its parameters, to obtain the optimal performance of network intrusion detection model, one needs to select the most adaptive RBF neural network parameters [11]. In current network intrusion detection model, feature selection and classifier parameters selection are separated, and independently implemented, so we have no idea about making feature selection first or RBF neural network parameter selection, and it is often identified by random, and thus difficult to get the optimal feature subset and RBF neural network parameters, to obtain the optimum effect of network intrusion detection, feature selection and parameters of RBF neural network should be done at the same time.

In order to improve the network intrusion detection accuracy, overcome the separate selection defect of traditional features and parameters of RBF neural network, it put forward the improved particle swarm optimization, IPSO to optimize the neural network network intrusion detection model(IPSO RBF). Simulation experiments using KDD Cup 99 data sets, to verify the effectiveness and superiority of IPSO- RBF network intrusion detection model.

\section{Feature Selection and RBF Neural Network Parameter Optimization}

\subsection{Feature Selection Optimization Problem}

For network status feature set $F=\left\{f_{1}, f_{2}, \ldots, f_{n}\right\}$, it adopts the binary vector to indicate feature selection as: $\mathrm{S}=\left\{\mathrm{s}_{1}, \mathrm{~s}_{2}, \ldots, \mathrm{s}_{\mathrm{n}}\right\}, \mathrm{s}_{\mathrm{i}} \in\{0,1\}, \mathrm{i}=1,2, \ldots, n$, where $\mathrm{n}$ is the network feature dimension, 1 and 0 respectively represent the corresponding feature is selected or not.

The purpose of feature selection is to reduce feature dimension, and improve the network intrusion accuracy, so the network state feature optimization problem can be described as:

$$
\begin{aligned}
& \max _{S} G(S) \\
& \text { s.t. } \\
& \left\{\begin{array}{l}
S=\left\{s_{1}, s_{2}, \cdots, s_{n}\right\} \\
s_{i} \in\{0,1\} \\
i=1,2, \cdots, n
\end{array}\right.
\end{aligned}
$$

\subsection{RBF Neural Network Parameter Optimization Problem}

RBF neural network is a kind of three-layer feed forward neural network, input layer connects network with outer environment, implicit layer makes mapping of signals between input and hiding layer, output layer outputs vector. The RBF neural network structure as shown in Figure 1, where R should be shown in $\mathrm{C}$

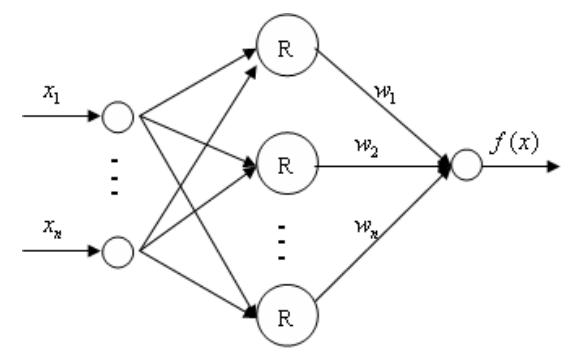

Figure 1. RBF Neural Network Structure 
Sets the input vector of the ith sample point as $X_{\mathrm{i}}(\mathrm{i}=1,2, \ldots, \mathrm{n})$, the output vector of $\mathrm{jth}$ sample point as $Y_{\mathrm{j}}(\mathrm{j}=1,2, \ldots, \mathrm{m})$, the $\mathrm{jth}$ radial basis function center vector as $C_{\mathrm{j}}\left(\mathrm{j}=1,2, \ldots, \mathrm{n}_{\mathrm{c}}\right)$, then the network output for RBF neural network as

$Y_{i j}=w_{i 0}+\sum_{k} w_{i k} f\left(\left\|x_{j}-c_{k}\right\|\right)=\sum_{k=0}^{n_{c}} w_{i k} f\left(c_{k}-x_{j}\right)$

In formula, wij is the weight between implicit layer and output node, $w_{\mathrm{i} 0}$ is the threshold of the ith node, $Y_{\mathrm{ij}}$ is the output ith node, $n_{c}$ is the number of implicit layer nodes, $\mathrm{f}$ is radial basis function, its definition as follows: formula (3) is wrong

$R_{i}(x)=\exp \left(\frac{\left\|x-c_{i}\right\|}{2 \sigma_{i}^{2}}\right) \quad i=1,2, \cdots, m$

Where, $\left\|\mathrm{x}-c_{\mathrm{i}}\right\|$ indicates the distance between $\mathrm{x}$ and $c_{\mathrm{i}}, c_{\mathrm{i}}$ indicates the center value of implicit function, $\sigma_{\mathrm{i}}$ represents core width.

RBF neural network performance value $w_{\mathrm{ij}}, c_{\mathrm{i}}$ and $\sigma_{\mathrm{i}}$, the values are interrelated, to obtain the optimal RF neural network model, one needs to select the most appropriate $w_{\mathrm{ij}}$ , $\quad c_{\mathrm{i}}, \quad \sigma_{\mathrm{i}}$ first, so RBF neural network parameter optimization model is:

$M=\left\{w_{i j}, c_{i}, \sigma\right\}$

Using the network intrusion detection accuracy $(\mathrm{G})$ as objective function parameters optimization neural network, RBF neural network parameters of the optimization problem can be described as:

$\max _{M} G(M)$

s.t.

$$
\left\{\begin{array}{l}
M=\left\{w_{i j}, c_{i}, \sigma_{i}\right\} \\
w_{i j}>0 \\
c_{i}>0 \\
\sigma_{i}>0
\end{array}\right.
$$

\subsection{Joint Optimization of Feature Selection and RBF Neural Network Parameter}

For the optimization objectives of formula(1) and (5) are improving the detection accuracy of network intrusion detection, so in order to make the network intrusion detection results with better accuracy, excavating the relation between feature subset selection and RBF neural network parameters, the feature subset selection and parameters of RBF neural network optimization should be done at the same time.

The mathematical model of feature selection and parameters of RBF neural network joint optimization as:

$\max _{S, M} G(S, M)$

Where, G represents network intrusion detection accuracy; S represents feature subset; $M$ represents RBF neural network parameters.

PSO algorithm is a kind of representative cluster intelligent algorithm simulated birds feeding behavior, through the information exchange between particles, mutual cooperation to find the optimal location, easy to implement and has strong parallelism and fast search capabilities, which is able to find the global optimal solution in a short time. Therefore, this study uses PSO algorithm to select feature subset and $w_{\mathrm{ij}}, c_{\mathrm{i}}, \sigma_{\mathrm{i}}$ parameter of RBF neural network, and make improvement on them, so as to improve the network intrusion detection accuracy. 


\section{Intrusion Detection Model of IPSO-RBF Neural Network}

\subsection{Improve Particle Swarm Optimization Algorithm (The Expression of Formula (7) is Error)}

Set $\mathrm{Xi}=\left[\mathrm{x}_{i, 1}, \mathrm{x}_{i, 2}, \ldots, x_{i d}\right]$ and $\mathrm{V}_{\mathrm{i}}=\left[\mathrm{v}_{i, l}, \mathrm{f}_{i, 2}, \ldots, v_{i d}\right]_{i}$ as the current position and velocity of particle $\mathrm{i}$, respectively, $P_{i=}\left[\mathrm{p}_{i, 1}, \mathrm{p}_{i, 2}, \ldots, p_{i d}\right]$ and $p_{g=}\left[\mathrm{p}_{g, 1}, \mathrm{p}_{g, 2}, \ldots, p_{g d}\right]$ are the best positions of particle $\mathrm{i}$ and particle swarm experienced respectively, particle velocity and position updating formula as

$$
\begin{aligned}
v_{i, j}(t+1) & =\omega v_{i, j}(t)+c_{1} \times r_{1} \times\left(p_{i, j}-v_{i, j}(t)\right) \\
c_{2} & \times r_{2} \times\left(p_{g, j}-x_{i, j}(t)\right) \\
x_{i, j}(t+1) & =x_{i, j}(t)+v x_{i, j}(t+1), j=1,2, \cdots, d
\end{aligned}
$$

Where, $c_{1}$ and $c_{2}$ are acceleration coefficient; $\mathrm{K}$ is the current iteration number; $\mathrm{R} 1$ and $\mathrm{R} 2$ are the random number within $[0,1]$ scope; $\omega$ is inertia weight.

If a local optimum position is found on a particle, other particles then quickly aggregate to it, thus the particle swarm are easy to be local optimum, and appear prematurity phenomenon.

Chaos has sensitivity to initial conditions, with strong traverse ability, can use this property for particle swarm chaos disturbance, so as to improve the search ability of particle swarm optimization algorithm. A typical Logistic equation as shown in formula(9), it can be seen form formula (9), the random initial value $z_{0}$ can iterate a certain time sequence $\mathrm{z}_{1}, \mathrm{z}_{2}, \mathrm{z}_{3}, \ldots$

$z_{i+1}=\mu z_{i}\left(1-z_{i}\right), i \in(0,1)$

Where, $\mu$ is control parameter.

Based on the overall change of particle swarm fitness to judge whether the population appears premature state or not. The fitness variance of particle swarm $\left(\sigma^{2}\right)$ can be defined as

$\sigma^{2}=\sum_{t=1}^{N}\left(\frac{f_{t}-f_{\text {avg }}}{f}\right)$

Where, $\mathrm{N}$ is particle swarm scale; $f_{\text {avg }}$ is the average fitness of particle swarm; $f$ is the normalized scaling factor; $f_{i}$ is the fitness of the individual $\mathrm{i}$.

\subsection{Network Intrusion detection procedure of IPSO-RBF Neural Network}

(1) Collect state information of network system, and transform it into the format can be identified by the RBF neural network.

(2) To generate initial particle swarm, particle bit string including network feature subset, RBF neural network parameters $w_{\mathrm{ij}}, c_{\mathrm{i}}, \sigma_{\mathrm{i}}$, according to the initial particle fitness value to determine individual extremum value $P_{t}$ and swarm extremum value $P_{g}$, and regard the adaptive optimal particle position as a global optimal solution.

(3) Decoding the bit string of the particle, and then according to the feature subset to process the training sample, input to the RBF neural network for training, parameters are $w_{\mathrm{ij}}, c_{\mathrm{i}}, \sigma_{\mathrm{i}}$, and calculate the particle fitness value according to the results of the test. Fitness function $f(x)$ are defined as follows

$f(x)=f(S, M)=\frac{\mathrm{p}}{\mathrm{t} \text { ot al }} \times 100 \%$

Where, $\mathrm{p}$ indicates the detected intrusion number; total indicates the total intrusion number, $\mathrm{S}$ indicates the selected feature subset, $\mathrm{M}$ indicates the selected $\mathrm{RBF}$ neural network parameters. 
(4) If the particle fitness value $\mathrm{f}(\mathrm{xi})$ is better than that of its historical optimal value $f\left(P_{\text {best }}, i\right)$, then the particle position to replace $f\left(P_{\text {best }}, i\right)$.S Similarly, if $f\left(x_{i}\right)>f\left(g_{\text {bes }}, i\right)$, then use the particle position instead of $f\left(g_{\text {best }}, i\right)$.

(5) According to formula (7) and (8) to update the speed and position of the particle, and produce a new generation of particle swarm $x(t+1)$.

(6) Calculate the fitness variance $\left(\sigma^{2}\right)$ of particle swarm, if the adjacent twice $\sigma^{2}$ difference are less than the threshold value of $\mathrm{C}$, then indicates a premature phenomenon, and turn to step7; otherwise to Step8.

(7) to carry out chaos disturbance on optimal position of particle swarm vector $p_{g=}\left[\mathrm{p}_{g, l}, \mathrm{p}_{g, 2}, \ldots, p_{g d}\right]$. Specific steps are as follows

(1) Let $\mathrm{p}_{\mathrm{g}}$ mapping to definitional domain [0.1] of formula (9) through the following equation.

$z_{i}=\frac{p_{g i}-a_{i}}{b_{i}-a_{i}}$

(2) $z_{i}^{(m)}(m=1,2, \cdots)$ 。 Through multiple iterations of Logistic equation $z_{i+1}=\mu z_{i}\left(1-z_{i}\right)$, and obtained chaos sequence: $z_{i}^{(m)}(m=1,2, \cdots)$.

(3)The generated value by formula (14) inverse mapping back to the original solution space, and generate a chaotic variables feasible solution sequence accordingly : $p_{g}^{(m)}=\left(p_{g 1}^{(m)}, p_{g 2}^{(m)}, \cdots, p_{g d}^{(m)}\right)$ 。

$p_{g i}^{(m)}=a_{i}+\left(b_{i}-a_{i}\right) a_{i}^{(m)}$

(4) Calculate the fitness value of feasible solution $p_{g}^{(m)}(m=1,2, \cdots)$ after chaos processing, and the optimal value $\mathrm{p} *$ of fitness value.

(5) To replace the random selected particle position with $p^{*}$ position vector.

(8) If the maximum number of iterations is reached, then return to the global optimal particle position, otherwise jump to step (3) for optimizing continuously.

(9) The optimal particle bit string will be decoded into network feature subset, RBF neural network parameters $w_{\mathrm{ij}}, \quad c_{\mathrm{i}}, \quad \sigma_{\mathrm{i}}$.

(10) To established the optimal network intrusion detection model based on network feature subset and parameters of RBF neural network $w_{\mathrm{ij}}, c_{\mathrm{i}}, \quad \sigma_{\mathrm{i}}$.

\section{Simulation Experiment}

\subsection{Data Sources}

To test the performance of network intrusion detection model of IPSO - RBF neural network, it uses KDD Cup 99 data sets, and carries out simulation experiment on Matlab 2009 software. As the mass samples in KDD Cup 99 data sets, so it randomly selects a portion of data for the test, the data distribution are shown in table 1.

Table 1. Experiment Samples Distribution

\begin{tabular}{ccc}
\hline Intrusion type & Training sample & Test sample \\
\hline DoS & 1200 & 300 \\
Probe & 2400 & 800 \\
R2L & 100 & 40 \\
U2R & 300 & 100 \\
\hline
\end{tabular}




\subsection{Comparison Model and Evaluation Index}

In order to make the IPSO - RBF neural network detection results comparable, with neither no feature selection, and random selection of RBF neural network (RBF) parameters. Only using PSO for feature selection of RBF neural network (PSO RBF1);no feature selection, only adopt parameters of RBF neural network (PSO - RBF2) optimized by PSO; using PSO for both feature selection and parameters of RBF neural network optimization, but the separated (PSO - RBF3) as comparison model. To evaluate the index collection and network intrusion detection accuracy.

\subsection{Pre-Processing of the Data}

In order to prevent the feature value over large or small to influence the speed of RBF neural network training, so it makes normalization processing on feature value and specified as

$$
x_{i}^{\prime}=\frac{x_{i}}{\max \left(x_{i}\right)}
$$

Where, $\max \left(\mathrm{x}_{\mathrm{i}}\right)$ is the maximum of feature $\mathrm{x}_{\mathrm{i}}, x_{i}$ 和 $x_{i}$ represents original and normalized features.

\subsection{Results and Analysis}

4.4.1. Comparison of Intrusion Detection Accuracy: Input all kinds of intrusion training samples into the RBF neural network for study, and make corresponding feature and parameter optimization, and establish the corresponding network intrusion detection model, and then do detection on test samples, the results of 10 times operation of each model on each data set, their average network intrusion detection accuracy (\%) as shown in 2 .

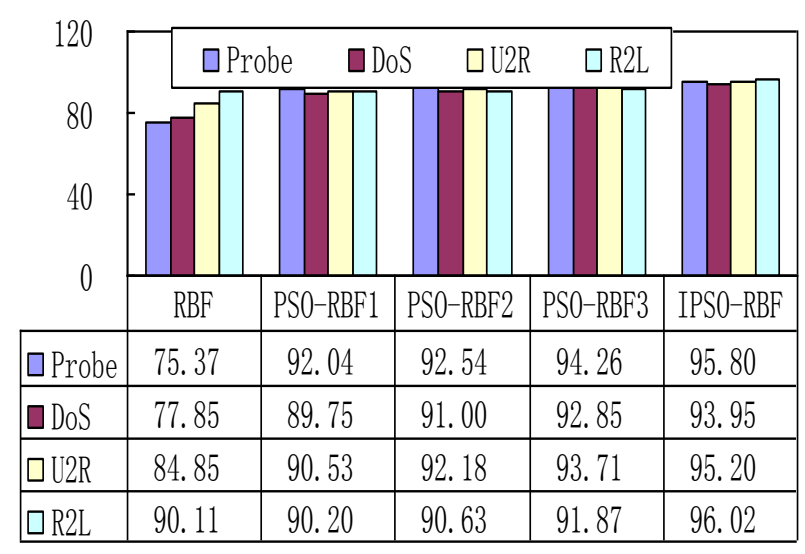

Figure 2. Accuracy Comparing Different Models of Intrusion Detection

The conclusions of the analysis of comparison results in Figure 2 are as follows:

(1) Relative to RBF neural network, the average detection accuracy of both feature selection and RBF neural network parameter optimization model are improved to some extent, this indicates that both feature subset selection and RBF neural network optimization can improve the network intrusion detection accuracy.

(2) The intrusion detection accuracy of IPSO -RBF neural network model is the highest, it shows that single feature subset selection or neural network parameters optimization, can optimize only one of its aspects, without considering the connection of both, while IPSO-RBF neural network make optimization on both feature selection and 
RBF neural network parameters at the same time, thus can make good description of network state information, and detect network intrusion behavior accurately.

4.4.2. Detection Time Comparison: Network real time capability is very important to network intrusion detection, the use of tic and toc command to record average detection time (s) of test sample of each model, and test results are shown in table 2.

Table 2. Detection Time Comparison of Different Model

\begin{tabular}{cccccc}
\hline Intrusion type & RBF & PSO-RBF1 & PSO-RBF2 & PSO-RBF3 & IPSO-RBF \\
\hline Probe & 16.25 & 12.22 & 11.06 & 9.80 & 6.17 \\
DoS & 11.87 & 9.23 & 8.88 & 6.06 & 5.20 \\
U2R & 4.42 & 2.79 & 2.58 & 1.92 & 1.46 \\
R2L & 2.79 & 2.03 & 1.28 & 1.13 & 1.06 \\
\hline
\end{tabular}

It is known from table 2, among all the network intrusion detection models, the detection time of IPSO - RBF is the shortest, which indicates its detection speed is the fastest, this is mainly due to the IPSO - RBF made optimization on feature selection and parameters of RBF neural network at the same time, thus effectively reduced the feature dimension and RBF neural network input node number, and the computational complexity was also reduced, so the network intrusion detection was speed up accordingly.

\section{Conclusion}

After fully considering the relation between feature subset selection and parameters of RBF neural network, it proposed the network intrusion detection model of IPSO - RBF neural network. The simulation results showed that, compared with traditional network intrusion detection model, IPSO- RBF neural network reduced the feature dimension, and obtained the better parameters of RBF neural network, and thus improved the effect of the network intrusion detection.

\section{Acknowledgements}

This work was supported by .Hunan Province Natural Science Foundation Project No. $14 J J 2124$.

\section{References}

[1] Lv Z, Tek A, Da Silva F. Game on, science-how video game technology may help biologists tackle visualization challenges[J]. PloS one, (2013), vol. 8, no. 3, p. 57990.

[2] Su T, Wang W, Lv Z. Rapid Delaunay triangulation for randomly distributed point cloud data using adaptive Hilbert curve[J]. Computers \& Graphics, (2016), no. 54, pp. 65-74.

[3] Lin Y, Yang J, Lv Z. A Self-Assessment Stereo Capture Model Applicable to the Internet of Things[J]. Sensors, (2015), vol. 15, no. 8, pp. 20925-20944.

[4] Yang J, Chen B, Zhou J. A Low-Power and Portable Biomedical Device for Respiratory Monitoring with a Stable Power Source[J]. Sensors, (2015), vol. 15, no. 8, pp. 19618-19632.

[5] Lv Z, Halawani A, Fen S. Touch-less Interactive Augmented Reality Game on Vision Based Wearable Device $[\mathrm{J}]$. Personal and Ubiquitous Computing, (2015).

[6] ] Gu W, Lv Z, Hao M. Change detection method for remote sensing images based on an improved Markov random field[J]. Multimedia Tools and Applications, (2015): pp. 1-16.

[7] Chen Z, Huang W, Lv Z. Towards a face recognition method based on uncorrelated discriminant sparse preserving projection[J]. Multimedia Tools and Applications, (2015), pp. 1-15.

[8] Jiang D, Ying X, Han Y. Collaborative multi-hop routing in cognitive wireless networks[J].Wireless Personal Communications, (2015), pp. 1-23.

[9] Lv Z, Halawani A, Feng S. Multimodal hand and foot gesture interaction for handheld devices[J]. ACM Transactions on Multimedia Computing, Communications, and Applications (TOMM), (2014), vol. 11, no. (1s), p. 10. 
[10] Li X, Lv Z, Hu J. XEarth: A 3D GIS Platform for managing massive city information[C]. Computational Intelligence and Virtual Environments for Measurement Systems and Applications (CIVEMSA), 2015 IEEE International Conference on. IEEE, (2015), pp. 1-6.

[11] ] Li X, Lv Z, Hu J. Traffic management and forecasting system based on 3d gis[J]. IEEE International Symposium on Cluster, Cloud and Grid Computing (CCGrid), (2015).

[12] Zhang S, Jing H. Fast log-Gabor-based nonlocal means image denoising methods[C]. Image Processing (ICIP), 2014 IEEE International Conference on. IEEE, (2014), pp. 2724-2728.

[13] Zhang S, Jing H. Fast log-Gabor-based nonlocal means image denoising methods[C]. Image Processing (ICIP), 2014 IEEE International Conference on. IEEE, (2014), pp. 2724-2728.

[14] Jiang $\mathrm{D}, \mathrm{Xu} \mathrm{Z}$, Chen Z. Joint time-frequency sparse estimation of large-scale network traffic[J]. Computer Networks, (2011), vol. 55, no. 15, pp. 3533-3547.

[15] Jiang D, Xu Z, Li W. An Energy-Ecient Multicast Algorithm with Maximum Network Throughput in Multi-hop Wireless Networks[J]. Journal of Communications and Networks. (2015).

[16] Liu Y, Yang J, Meng Q. Stereoscopic Image Quality Assessment Method based on Binocular Combination Saliency Model[J]. Signal Processing. (2015).

[17] Yang J, He S, Lin Y. Multimedia cloud transmission and storage system based on internet of things. Multimedia Tools and Applications. (2016).

[18] Li T, Zhou X, Wang K. A convergence of key-value storage systems from clouds to supercomputers[J]. Concurrency and Computation: Practice and Experience, (2015).

[19] Jiang D, Ying X, Han Y. Collaborative multi-hop routing in cognitive wireless networks[J]. Wireless Personal Communications, (2015), pp. 1-23.

[20] X Song, Y Geng, Distributed community detection optimization algorithm for complex networks, Journal of Networks, vol. 9, no. 10, pp. 2758-2765, Jan. (2014).

[21] Lv Z, Halawani A, Feng S. Multimodal hand and foot gesture interaction for handheld devices[J]. ACM Transactions on Multimedia Computing, Communications, and Applications (TOMM), (2014), 11(1s): 10.

[22] G Liu, Y Geng, K Pahlavan, Effects of calibration RFID tags on performance of inertial navigation in indoor environment, 2015 International Conference on Computing, Networking and Communications (ICNC), Feb. (2015).

[23] J He, Y Geng, Y Wan, S Li, K Pahlavan, A cyber physical test-bed for virtualization of RF access environment for body sensor network, IEEE Sensor Journal, 13(10), 3826-3836, Oct. (2013).

[24] Wenhua Huang, Yishuang Geng, Identification Method of Attack Path Based on Immune Intrusion Detection, Journal of Networks, vol. 9, no. 4, pp. 964-971, Jan. (2014).

[25] Li X, Lv Z, Hu J. XEarth: A 3D GIS Platform for managing massive city information[C]. Computational Intelligence and Virtual Environments for Measurement Systems and Applications (CIVEMSA), 2015 IEEE International Conference on. IEEE, (2015), pp. 1-6.

[26] J He, Y Geng, F Liu, C Xu, CC-KF: Enhanced TOA Performance in Multipath and NLOS Indoor Extreme Environment, IEEE Sensor Journal, vol. 14, no. 11, pp. 3766-3774, Nov. (2014).

[27] N Lu, C Lu, Z Yang, Y Geng, Modeling Framework for Mining Lifecycle Management, Journal of Networks, vol. 9, no. 3, pp. 719-725, Jan. (2014).

\section{Authors}

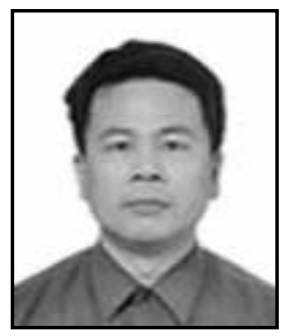

WANG Xingzhu. WANG Xingzhu, male, was born in 1974, in Hunan province. Now, he is an associate professor in Furong College Hunan, University of Arts and Science. His Main research area is network security. 